\title{
Decorated Attribute Grammars: Attribute Evaluation Meets Strategic Programming
}

\author{
Lennart C.L. Kats ${ }^{1}$, Anthony M. Sloane ${ }^{1,2}$, and Eelco Visser ${ }^{1}$ \\ ${ }^{1}$ Software Engineering Research Group, Delft University of Technology, \\ The Netherlands \\ L.C.L.Kats@tudelft.nl, visser@acm.org \\ 2 Department of Computing, Macquarie University, \\ Sydney, Australia \\ Anthony.Sloane@mq.edu.au
}

\begin{abstract}
Attribute grammars are a powerful specification formalism for treebased computation, particularly for software language processing. Various extensions have been proposed to abstract over common patterns in attribute grammar specifications. These include various forms of copy rules to support non-local dependencies, collection attributes, and expressing dependencies that are evaluated to a fixed point. Rather than implementing extensions natively in an attribute evaluator, we propose attribute decorators that describe an abstract evaluation mechanism for attributes, making it possible to provide such extensions as part of a library of decorators. Inspired by strategic programming, decorators are specified using generic traversal operators. To demonstrate their effectiveness, we describe how to employ decorators in name, type, and flow analysis.
\end{abstract}

\section{Introduction}

Attribute grammars are a powerful formal specification notation for tree-based computation, particularly for software language processing [26], allowing for modular specifications of language extensions and analyses. At their most basic, they specify declarative equations indicating the functional relationships between attributes (or properties) of a tree node and other attributes of that node or adjacent parent and child nodes [19]. An attribute evaluator is responsible for scheduling a tree traversal to determine the values of attributes in a particular tree.

Attribute grammars are nowadays employed in a wide range of application domains and contexts. To extend their expressiveness for use in particular domains, and to abstract over commonly occurring patterns, basic attribute grammars have been extended in many ways, in particular supporting attribution patterns with non-local dependencies. For example, remote attribution constructs allow equations that refer to attributes of nodes arbitrarily far above or below the node for which they are defined [5]15]. Chain attributes express a dependence that is threaded in a left-to-right, depth-first fashion through a sub-tree that contains definitions of the chain value [15]. Self rules provide a local copy of subtrees, which may be adapted for tree transformations [2]. More generally, collection attributes enable the value of an attribute of one node to be determined

O. de Moor and M. Schwartzbach (Eds.): CC 2009, LNCS 5501, pp. 142 157, 2009.

(c) Springer-Verlag Berlin Heidelberg 2009 
at arbitrary other nodes [5]22]. A different kind of remote attribute is provided by reference attribute grammars that allow references directly to arbitrary non-local nodes and their attributes [12], allowing for attributes that look up a particular node or collection of nodes. Finally, some attribute grammar systems support equations with circular dependencies that are evaluated to a fixed point [423].

All of these extensions aim to raise the level of abstraction in specifications, by translation into basic attribute grammars or by using an extended evaluator. Unfortunately, each of these extensions has been designed and implemented separately and is hardwired into a particular attribute grammar system.Potential users may find that a particular system does not provide the set of demanded extensions. Adding new abstractions is non-trivial, since it requires modification of the attribute evaluation system itself.For example, it can sometimes be useful to thread attribute values from right-to-left (e.g., when computing backward slices or use-def relations between variables). In a system with only left-to-right chained attributes, this dependence must be encoded using basic attribute equations, despite the similarity of the abstractions.

In his OOPSLA'98 invited talk, "Growing a Language" [28], Guy Steele argued that "languages need to put the tools for language growth in the hands of the users," providing high-level language features that abstract over various extensions, rather than directly providing language features to solve specific problems. To this effect, we propose attribute decorators as a solution for the extensibility problem of attribute grammar specification languages. A decorator is a generic declarative description of the tree traversal or evaluation mechanism used to determine the value of an attribute. Decorators augment basic attribute equations with additional behavior, and can provide nonlocal dependencies or a form of search as required. For instance, a decorator can specify that the value of an attribute is to be sought at the parent node (and recursively higher in the tree) if it is not defined at the current node. Decorators can also enhance the usability of attribute equations for specific domains, separating the generic behavior from specific equations such as type checker constraints or data-flow equations, supported in other systems through specialized extensions.

In this paper, we present ASTER, a system for decorated attribute grammars (available from [1]). Decorators are powerful enough to specify all of the attribute grammar extensions listed above, avoiding the need to hardwire these into the system. A library of decorators suffices to cover common cases, while user-defined, domain-specific decorators can be used for specific applications and domains.

Decorators are inspired by strategic programming, where generic traversal strategies enable a separation between basic rewrite rules defining a tree transformation and the way in which they are applied to a tree [33|20|21]. In our case, local attribute equations define the core values of a tree computation, while decorators describe how those values are combined across the tree structure. The ASTER specification language is built as an extension of the Stratego strategic programming language [8]. We reuse the generic traversal operators of Stratego for the specification of decorators, and its pattern matching and building operations as the basis for attribute equations.

We begin this paper with background on attribute grammars and introducing our basic notations. Section 3 defines decorators, showing how they augment basic equations and capture common patterns. In Section 4 we present typical language engineering 
applications, demonstrating how decorators can be effectively applied in this area. We briefly outline our implementation in Section 5. Finally, we conclude with a comparison to related work and some pointers to future directions.

\section{Attribute Grammars}

As they were originally conceived, attribute grammars (AGs) specify dependencies between attributes of adjacent tree nodes [19]. Attributes are generally associated with context-free grammar productions. For example, consider a production $\mathrm{X}::=\mathrm{Y} \mathrm{Z}$. Attribute equations for this production can define attributes for symbols X, Y and Z. Attributes of $\mathrm{X}$ defined at this production are called synthesized, as they are defined in the context of $X$. They can be used to pass information upwards. Conversely, attributes of $Y$ and $\mathrm{Z}$ defined in this context can be used to pass information downwards, and are called inherited attributes.

\subsection{Pattern-Based Attribute Grammars}

In this paper we adopt a notational variation on traditional AGs in which attribute equations are associated with tree or term patterns instead of grammar productions [106]. Trees can be denoted with prefix constructor terms such as Root(Fork (Leaf (1), Leaf (2))). Tree patterns for matching and construction are terms with variables (indicated in italics throughout this paper), e.g. Fork $\left(t_{1}, t_{2}\right)$.

Basic attribute equations have the form

eq $p: r . a:=v$

and define equations for a term that matches pattern $p$, where attribute $a$ with a relation $r$ to the pattern has value $v$. The relation $r$ can be a subterm of $p$ indicated by a variable or the term matched by the pattern itself, indicated by the keyword id.

As an example, consider the transformation known as Bird's repmin problem [3], which can be well expressed as an AG, as illustrated in Figure 1 In this transformation, a binary tree with integer values in its leaves is taken as the input, and a new tree with the same structure and its leaves replaced with the minimum leaf value is produced as the output. For example, the tree Root (Fork (Leaf (1), Leaf (2))) is transformed to Root (Fork (Leaf (1), Leaf (1))).

In the specification of Figure 1, the local minimum leaf value in a subtree is computed in the synthesized attribute min (lines 3,8 and 12). At the top of the tree, the minimum for the whole tree is copied to the inherited global-min attribute (line 2), which is then copied down the tree to the leaves (lines 6 and 7). Finally, the replace attribute constructs a tree where each leaf value is replaced by the global minimum (lines 4, 9, 13).

Attribute equations are often defined in sets that share a common pattern, but may also be grouped to define a common attribute, which can make it easier to show the flow of information at a glance. Consider Figure 2 which is equivalent to the specification in Figure 1, but organizes the equations per attribute instead. Equations can be defined in separate modules, across different files, and are automatically assembled into a complete specification. Thus, language definitions can be factored per language construct and/or per attribute to support modular, extensible language definitions [13/31]. 


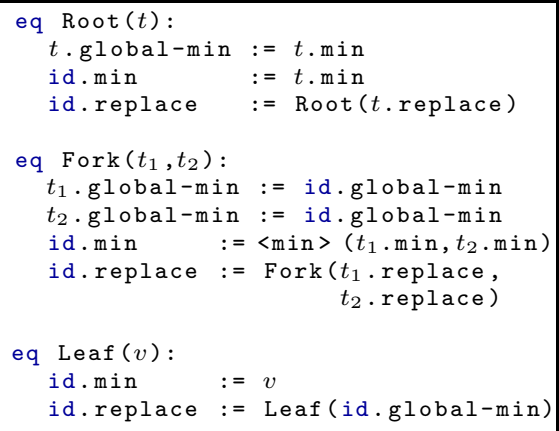

Fig. 1. An attribute grammar specification for repmin in pattern major form

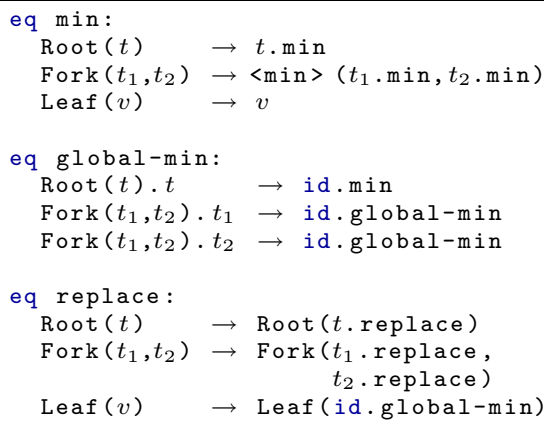

Fig. 2. An attribute grammar specification for repmin in attribute major form

Using patterns helps separation of concerns when specifying a syntax and AG analyses. However, it can still be useful to use the concrete syntax of a language. ASTER supports this using the generic approach of concrete object syntax embedding as described in [32]. For example, instead of a pattern While $(e, s)$, we can use a concrete syntax pattern, which is typically enclosed in "semantic braces":

eq | [ while (e) $s$ ] | :

id. condition $=e$

Concrete syntax patterns are parsed at compile-time, and converted to their abstract syntax equivalents. Section 4 includes further examples of this technique.

\subsection{Copy Rules}

In theory, basic attribute equations with local dependencies are sufficient to specify all non-local dependencies. Non-local dependencies can be encoded by passing context information around using local inherited and synthesized attributes. In the repmin example, this pattern can be seen in the definition of the global minimum value, which is defined in the root of the tree. This information is passed down by means of so-called copy rules, equations whose only purpose is to copy a value from one node to another.

To accommodate for the oft-occurring pattern of copying values through the tree, many AG systems provide a way to broadcast values, eliminating the need for tedious and potentially error-prone specification of copy rules by hand. For example, the repmin example can be simplified using the including construct of the GAG and LIGA systems [15], which provide a shorthand for specifying copy rules. Using this construct, the copy rules in Figure 11, lines 6 and 7 could be removed and line 13 replaced by id.replace := Leaf (including Root.global-min), specifying that the value is to be copied downward from the Root node.

\section{Decorators}

While constructs such as including provide notational advantages for some specifications, they cannot be used if the desired pattern of attribution does not precisely fit their 
definition. These notations are built into AG systems, and as such a developer is faced with an all-or-nothing situation: use a nice abstract notation if it fits exactly or fall back to writing verbose copy rules if there is no suitable shorthand. This section proposes attribute decorators as a more flexible alternative to building these shorthand abstractions into the AG system. Decorators can be defined to specify how attribute values are to be propagated through the tree. Common patterns such as including can be provided in a decorator library, while user-defined decorators can be written for other cases.

To define high-level attribute propagation patterns, we draw inspiration from strategic programming [33,20|21]. This technique allows the specification of traversal patterns in a generic fashion, independent of the structure of a particular tree, using a number of basic, generic traversal operations.

\subsection{Basic Attribute Propagation Operations}

Consider the specification of Figure 3. It specifies only the principal repmin equations, avoiding the copy rules. The flow of information is instead specified using decorators (at the top of the specification). For instance, global-min uses the down decorator, which specifies that values should be copied downwards. Before we elaborate on the decorators used in this example, let us first examine the unabbreviated set of equations and reduce them to a more generic form that uses elementary propagation operations. After this, we will show how these operations can be used in the specification of decorators.

Downward propagation of the global-min attribute, first defined at the root of the tree (as seen in Figure 3), was originally achieved by

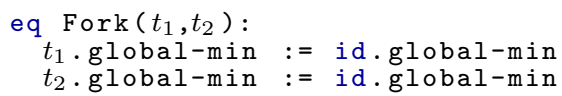

Another reading of this specification says that 'the global-min of any non-root term is the global-min of its parent.' Thus, if we can reflect over the tree structure to obtain the parent of a node, we can express this propagation as

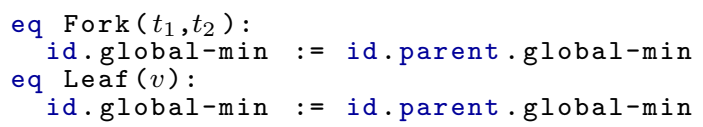

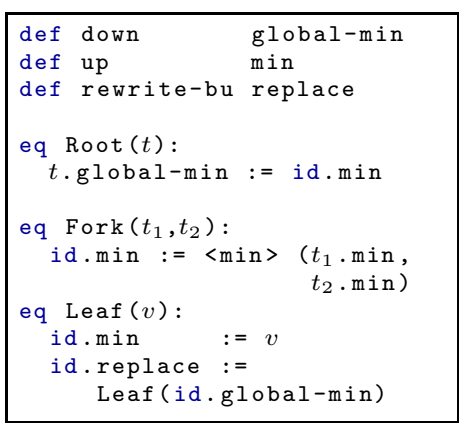

Fig. 3. Repmin using decorators

This notation makes the relation to the parent node's attribute value explicit, rather than being than implied by the context. It forms the basis of specifying the downward propagation in a more generic way: id.parent.global-min could be used as the default definition of global-min, used for nodes where no other definition is given (here, all non-root nodes). This is essentially what the down decorator in Figure 3 does.

A different form of propagation of values was used in the replace attribute:

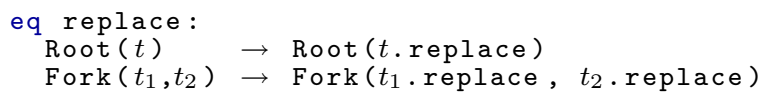

Here we can recognize a (common) rewriting pattern where the node names remain unchanged and all children are replaced. We abstract over this using the all operator: 


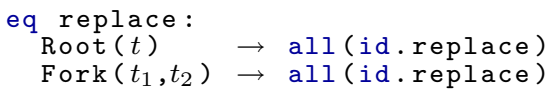

$\mathrm{all}$ is one of the canonical generic traversal operators of strategic programming [3320]. It applies a function to all children of a term. Other generic traversal operators include one, which applies a function to exactly one child, and some, which applies it to one or more children. In this case, we pass all a reference to the replace attribute. This reveals an essential property of attribute references in ASTER: they are first-class citizens that can be passed as the argument of a function in the form of a closure. The expression id.replace is a shorthand for a closure of the form $\lambda t \rightarrow(t$.replace). It can be applied to the current term in the context of an attribute equation or in a sequence of operations, or to a term $t$ using the notation $\langle f\rangle t$.

\subsection{Attribute Propagation Using Decorators}

We implement attribute definitions using functions that map terms to values. Parts of such a function are defined by attribute equations. Some attribute definitions form only a partial function, such as those in Figure 3. In that figure, copy rules are implicitly provided using decorators. Decorators are essentially higher-order functions: they are a special class of attributes that take another attribute definition (i.e., function) as their argument, forming a new definition with added functionality. This means that the declaration def down global-min and the accompanying equations for the global-min attribute effectively correspond to a direct (function) call to decorator down:

eq $\operatorname{Root}(t)$ :

$t$.global-min := id.down(the original global-min equations, here t.min)

A basic decorator $d$ decorating an attribute $a$ is specified as follows:

decorator $d(a)=s$

The body $s$ of a decorator is its evaluation strategy, based on the Stratego language [8]. It provides standard conditional and sequencing operations. Using generic traversal operators, the evaluation strategy can inspect the current subtree. These operators are agnostic of the particular syntax used, making decorator definitions reusable for different languages. In this paper, we introduce the notion of parent references as an additional generic traversal operator, in the form of the parent attribute. Furthermore, we provide a number of generic tree access attributes that are defined using these primitives, such as the prev-sibling and next-sibling attributes to get a node's siblings, and child $(c)$ that gets the first child where a condition $c$ applies. Finally, we introduce reflective attributes that provide information about the attribute being decorated. These include the defined attribute, to test if an attribute equation is defined for a given term, and the name and signature attributes to retrieve the attribute's name and signature.

To illustrate these operations, consider the definition of the down decorator, which defines downward propagation of values in the tree (see Figure 4). This decorator automatically copies values downwards if there is no attribute equation defined for a given node. It checks for this condition by means of the defined reflective attribute (1). In case there is a matching equation, it is simply evaluated (2). Otherwise, the decorator acts as a copy rule: it "copies" the value of the parent. For this it recursively continues 


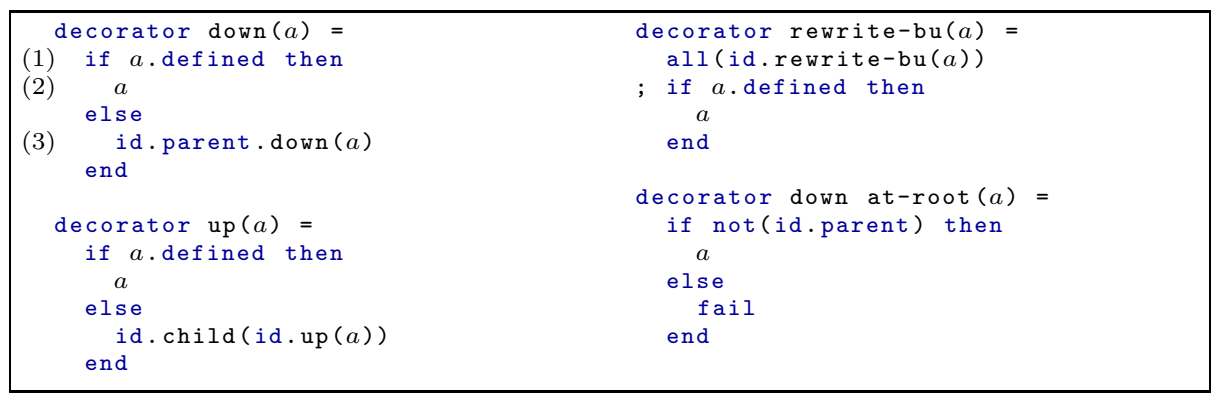

Fig. 4. Basic decorator definitions

evaluation at the parent node (3). Conversely, the up decorator provides upward propagation of values. If there is no definition for a particular node, it inspects the child nodes, eventually returning the first successful value of a descendant node's attribute equation.

The rewrite-bu decorator provides bottom-up rewriting of trees, as we did with the replace attribute. Using the all operator, it recursively applies all defined equations for an attribute, starting at the bottom of the tree. Rewrites of this type produce a new tree from an attribute, which in turn has attributes of its own, potentially allowing for staged or repeated rewrites.

In the next section we provide some examples of more advanced decorators. At their most elaborate, these may specify a pattern $p$, can be parameterized with functions $a *$ and values $v *$, and may themselves be decorated $(d *)$ :

decorator $d *[p$.$] name \left(d[, a *]\left[\begin{array}{l}\mid l \\ \text { d }\end{array}\right]\right)=s$

Note in particular the vertical bar ' $I$ ', used to distinguish function and value arguments; in a call $\mathrm{f}(\mid x), x$ is a value argument, in a call $\mathrm{f}(x)$ it is a function. The same convention, based on the Stratego notation, is supported for attributes. Furthermore, note that decorators can import other decorators $d *$. Such decorators are said to be stacked, and provide opportunity for reuse. To illustrate this, consider the at-root decorator of Figure 4 It evaluates attribute equations at the root of a tree, where the current node has no parent. Using the down decorator, the result is propagated downwards. Effectively, applying this stacked decorator results in a function application of the form id. down (id. at-root $(a)$ ). Stacking can also be achieved by declaring multiple decorators for an attribute. For example, we can add a "tracing" decorator to the global-min attribute, logging all nodes traversed by the down decorator:

def down trace global-min

\section{Applications}

In this section we discuss a number of common idioms in AG specifications, and show how attribute decorators can be used to encapsulate them. We focus on language processing, a common application area of AG systems. As a running example we use a simple "while" language (see Figure 5). We demonstrate different language analysis problems and how they can be dealt with using high-level decorators that are agnostic 


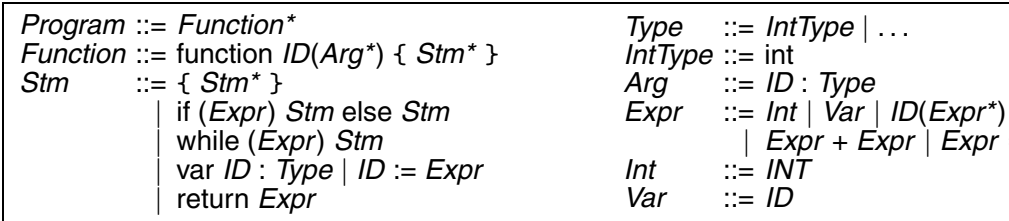

Fig. 5. The "while" language used in our examples

of the object language. As such, they are reusable for more sophisticated languages and other applications.

\subsection{Constraints and Error Reporting}

A fundamental aspect of any language processing system is reporting errors and warnings. We define these as declarative constraints using conditional attribute equations. These equations specify a pattern and a conditional where clause that further restricts the condition under which they successfully apply:

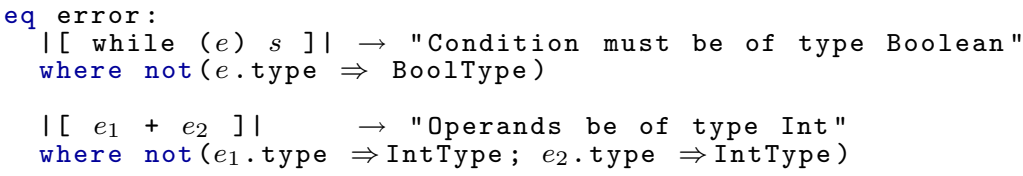

Each equation produces a single error message string if the subexpression types do not match IntType or BoolType. Rather than having them directly construct a list, we can collect all messages using the collect-all decorator (see Figure 6). It traverses the tree through recursion, producing a list of all nodes where the attribute succeeds. Note that this decorator does not test for definedness of the equations (using $a$.def ined), but rather whether they can be successfully applied. Using collect-all with the error attribute, we can define a new errors attribute:

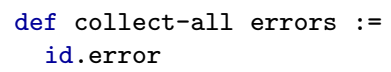

This notation both declares the decorators and a default equation body, which refers to error.

To provide usable error messages, however, the error strings need further context information. We can define a

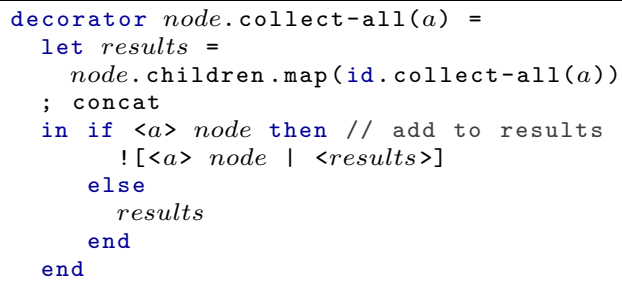

Fig. 6. The collect-all decorator new, application-specific decorator to add this information before they are collected, and use it to augment the error attribute:

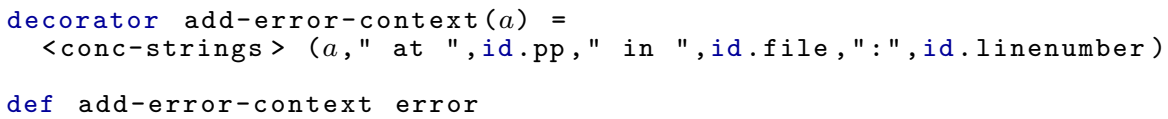


With this addition, the errors attribute now lists all errors, including a pretty-printed version of the offending construct (provided a pp attribute is defined), and its location in the source code (given a file and linenumber attribute).

\subsection{Name and Type Analysis}

Type analysis forms the basis of static error checking, and often also plays a role in code generation, e.g. for overloading resolution. Types of expressions typically depend on local operands, or are constant, making them well-suited for attribute equations. Moreover, an AG specification of a type analysis is highly modular, and may be defined across multiple files. Thus, let us proceed by defining a type attribute for all expressions in our language to perform this analysis:

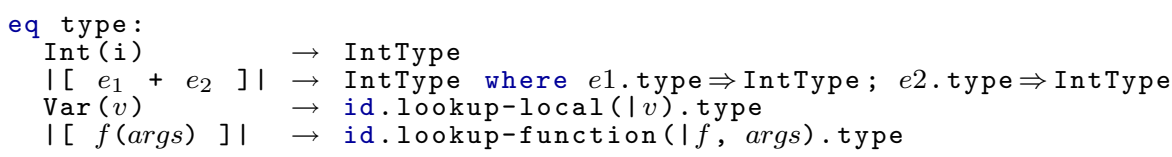

Variable references and function calls require non-local name analysis to be typed. This can be done using parameterized lookup attributes that given a name (and any arguments), look up a declaration in the current scope [12]. In the example we reference the local type attribute of the retrieved node, but lookup attributes can be used to access arbitrary non-local attributes for use in various aspects of the system. The actual lookup mechanism is provided by means of reusable decorators: to do this for a particular language, it suffices to select an appropriate decorator and define the declaration sites and scoping constructs of the language. Our lookup attributes are defined as follows:

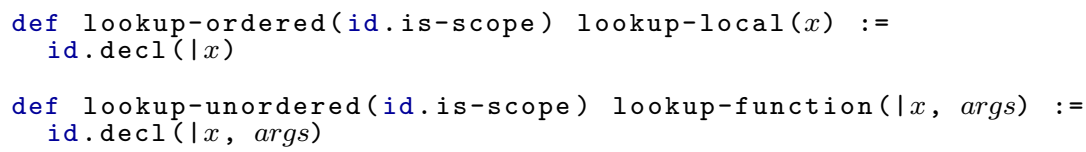

Figure 7 shows the prerequisite decl and is-scope attribute definitions for the name analysis, specified as arguments of the above attributes. Again, these are highly declarative and each address a single aspect. Declaration sites are identified by the decl attribute, which is parameterized with an identifier name $x$ and optionally a list of arguments. It only succeeds for matching declarations. All declarations also define a type attribute. Similarly, the is-scope attribute is used to identify scoping structures. Note in particular the equations of the "if" construct, which, for the purpose of this example, defines scopes for both arms, similar to try/catch in other languages.

Languages employ varying styles of scoping rules. In our language we have two kinds of scoping rules: C-like, ordered scoping rules, and Algol-like, unordered scoping rules. In many languages, local variables typically use the former, while functions typically use the latter. We define the lookup-ordered and lookup-unordered decorators to accommodate for these styles (see Figure 8). They traverse up the tree, inheriting the behavior of the down decorator, thus giving precedence to innermost scopes. Along this path, the lookup-ordered decorator visits the current node (1). If no declaration is found there (i.e., fetch-decl fails), the <+ combinator specifies that it should proceed at $(2)$, visiting any preceding siblings using the helper function lookup-outside-scopes. This function performs a local lookup for declarations in 


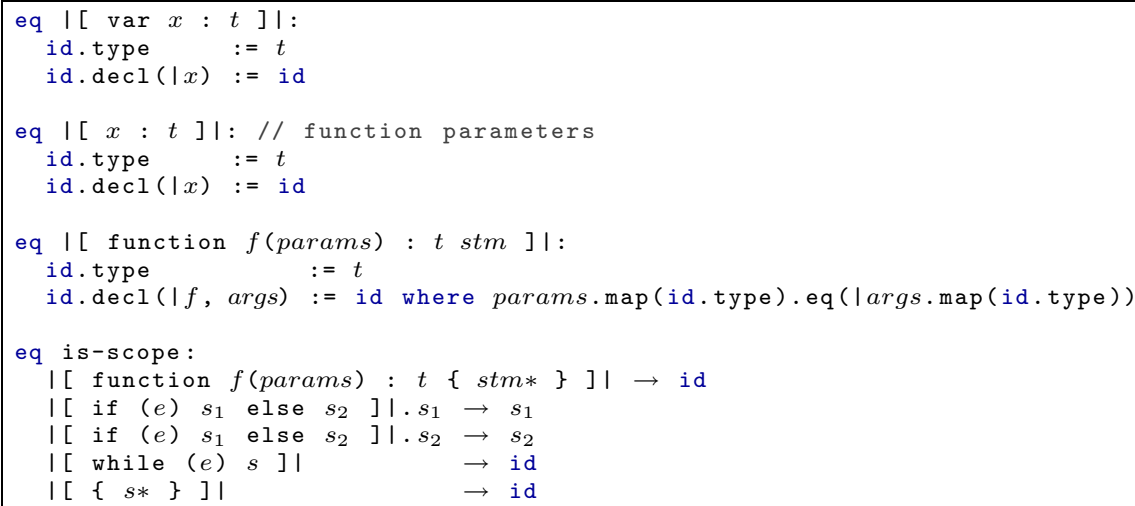

Fig. 7. Attributes for name analysis and types of declarations

these nodes, respecting the scoping rules by avoiding traversal of scoping constructs $(3)$. In contrast, lookup- unordered follows a straight path to the root of the tree, doing a search in encountered scopes (4).

\subsection{Flow Analysis}

Control-flow analysis forms the foundation of data-flow analysis, which is prerequisite to various compiler optimizations, refactorings, and static checks for bug patterns or security violations. A recent paper by Nilsson-Nyman et al. [25] demonstrated how AGs can be employed for modularly specifying such analyses, ensuring separation of concerns and reusability with different data-flow analyses.

We take an approach similar to that of the JastAdd project, using reference attributes [12] to declaratively define the control flow graph. Consider Figure 9] which defines a succ attribute, providing a reference to all successors of a statement. For instance, for the "if" statement, the successors are the "then" and "else" branches (1).

A helper attribute, succ-enclosing, determines the default successors based on the enclosing block. For sequences of statements, the successor is the next statement in the

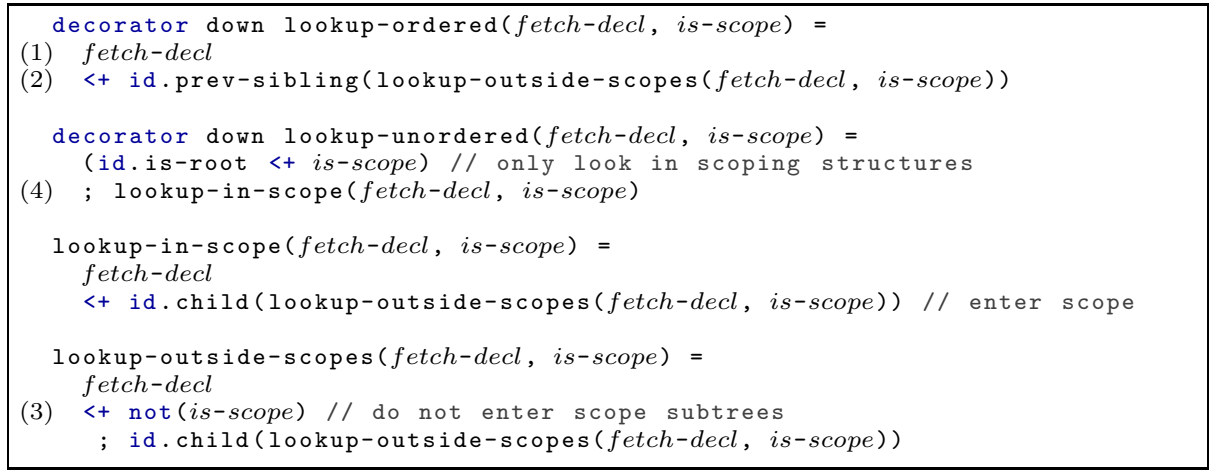

Fig. 8. Lookup attributes and decorators 
sequence (2). The "while" statement overrides this behavior, by setting the successor of the enclosed block to itself (3). For any non-control flow statements, we specify succ-enclosing as the default successor succ (4), using the default decorator (5).

The specification of the succ attribute allows for a natural, declarative way of specifying the forward control flow of a language. However, a number of data-flow analyses depend on the predecessors of a statement. To avoid specifying these by hand, it is possible to use collection attributes [5|22|4] to

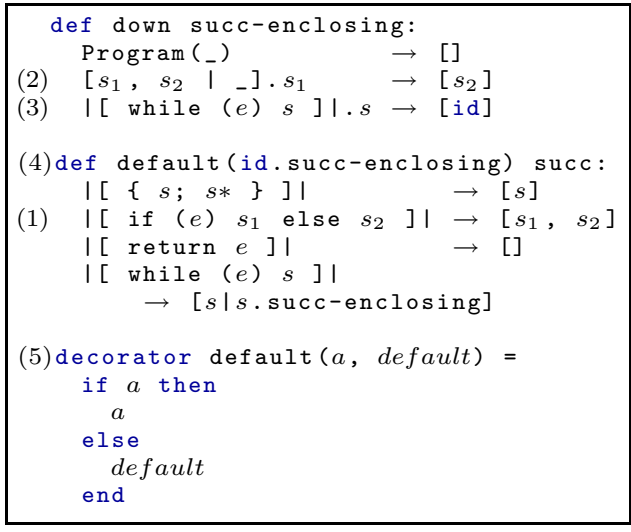

Fig. 9. Specification of the control flow derive the reverse flow graph. Collection attributes introduce a "contributes to" clause, allowing nodes to contribute values to collections in other nodes. Using this technique, we can define the predecessor graph in a single equation, by contributing each statement to its successors:

def contributes-to(id.succ) stm:

id.pred := stm

Figure 10 defines the contributes-to decorator. Note that for clarity, we use fragments of pseudocode in lieu of more advanced Stratego constructs. The complete, 20line source is available from [1]. This decorator operates in two phases: the first time any collection attribute is evaluated, it enters the survey phase (1), where the complete tree is traversed, adding all contributing nodes to a list maintained for each node contributed to. This is done only once, rather than for every collection attribute retrieved. After this phase completes (2), referenced collections only require the application of any attribute equations associated with it (for pred, stm is returned). Note that all required bookkeeping operations (i.e., storing contributions and whether the survey phase completed) are performed in the context of the current attribute: they are stored in tables associated with the attribute's unique signature and its argument values (i.e., id.signature).

The control flow graph, specified by the succ and pred attributes, forms the foundation of any data-flow analysis. As such a graph may have cycles in it, these analyses have the peculiar property that their equations may involve circular dependencies. This makes them unsuitable for traditional AGs. However, by extending the formalism with

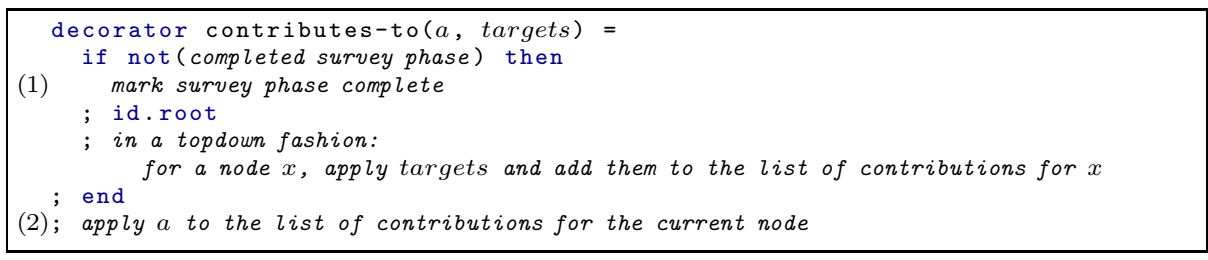

Fig. 10. The contributes-to decorator, contributing values to a list of nodes 
circular attributes [234], it becomes possible to use declarative AG equations to specify such analyses [25]. Circular attribute equations can be solved by fixed point iteration, as long as their underlying data forms a lattice. We implemented this in a decorator that evaluates circular attributes. However, due to a lack of space to fully explain the rather intricate algorithm that underlies it (see [234]), we do not include it here, and refer the interested reader to the technical report that accompanies this paper [17].

\section{Implementation}

The ASTER language is built as an extension of the Stratego strategic programming language [8], which natively supports the canonical generic traversal operators. The ASTER compiler is implemented in standard Stratego, using only a (bootstrapped) AG specification for error reporting (using constraint rules similar to those in Section 4.1). It compiles AG specifications to regular Stratego programs through a series of normalization steps. The normalization process starts by grouping attribute equations together, forming separate strategies for each attribute and decorator. As illustrated in Section 3.2 attribute equations and decorators are implemented as functions with generic traversal operations (called strategies in strategic programming). Inherited attributes are defined at the parent of a node; therefore, their implementation uses the parent primitive. Attribute references and imported decorators are converted to strategy calls. For decorator calls, static reflective data is added for reflective attributes such as signature. Finally, a memoization mechanism is added to cache all attribute and decorator calls. In the technical report that accompanies this paper, we elaborate on these normalization steps, using the repmin specification as an example [17].

Using memoization, attributes are evaluated at most once, thus achieving optimal evaluation. Similar memoization-based dynamic evaluation has been used before in many other systems, e.g. by Jalili [14] and recently in JastAdd [13]. In ASTER, memoization can be selectively disabled and overridden with custom behavior using decorators. For example, we disable it for fixpoint evaluation of data-flow equations.

Our current, experimental implementation has not been tuned for performance. One constraining factor is currently the ATerm library used to represent trees, which forms an integral part of Stratego. It is optimized for a maximally shared representation of terms, where identical subtrees occupy the same space in memory [7]. This makes it less suitable for storing additional, dynamic information in tree nodes, in our case parent references (for id.parent) and memoized attribute values. We worked around this by annotating tree nodes with unique keys, and use these to store the added information in separate tables. In the future, we would like to adapt or replace the underlying implementation to better accommodate for this. Regardless, preliminary performance measurements indicate promising results. We compared our compiler against JastAdd [13], a mature AG system that uses an evaluation mechanism conceptually very similar to our own. We used the repmin program of Figure 1 as a test case. Over an average of fifty runs, JastAdd took $51 \mathrm{~ms}$ to replace all leaves in a large tree with $2^{16}$ leaves. Our system took $150 \mathrm{~ms}$, or $180 \mathrm{~ms}$ for the version of Figure 3 where decorators are used in place of manual copy rules. Further testing confirms an unfortunate, but constant overhead of about a factor three in the base performance level, due to the expensive memoization and term initialization operations. Still, the results indicate a low overhead of the 
decorator mechanism. Furthermore, both our specifications, especially when using decorators, are more concise than the version implemented in JastAdd.

\section{Related Work}

The general principle behind attribute decorators shares similarities with the Decorator design pattern, which describes how to add functionality to objects at run-time [11]. Variations of this idea exist in languages such as Python, which features decorators for functions [27]. In our case, we augment basic attribute definitions with either propagation of values from other nodes or with higher-level behavior such as a circular evaluation scheme. This kind of augmentation is similar to code weaving used in many forms of aspect-oriented programming [18].

Although considerable research has been devoted to various special-purpose extensions of AGs (as illustrated in the preceding sections), rather less attention has been paid to extensibility of AG systems. Two systems that do aim at different degrees of extensibility are Silver [29] and first-class attribute grammars [24].

In first-class AGs, attribute equations are first-class citizens, allowing them to be combined and manipulated using the language itself. Using function combinators, basic basic up, down, and chain copy rules can be defined [24]. These combinators show similarities with decorators, although they are purely defined in terms of functional dependencies, and lack the reflective and traversal primitives that form the building blocks of decorators. The paper does not indicate that they could be used to implement more sophisticated forms of propagation and manipulation of equations, such as the collection and circularity decorators. Based on the Haskell type checker, first-class AGs prevent errors where the use of an attribute does not match its type. Errors due to cyclic dependencies or a mismatch between attribute equations and grammar productions are not reported. Our system is based on Stratego, which is largely untyped (but could be typed [21]). Further complicated by the use of parent node references, it currently does not provide a fully typed system, other than basic static pattern coverage checking.

Silver supports extension with automatic copy rules as well as more advanced features such as collection attributes in a relatively accessible manner [29]. Implemented in itself, the Silver language can be used to modularly implement such extensions. While adding extensions of this kind is made easier through facilities such as forwarding for local transformations [30] and higher-order attributes, it is hard to imagine a regular Silver user building such an extension. Moreover, it is difficult to encapsulate these extensions in a single application or library, as they must be integrated in the base AG system. In contrast, many decorators are light-weight so they can be developed quickly and easily as needed.

A system that particularly inspired our design has been JastAdd [13], which extends traditional AGs in a number of interesting ways 1 JastAdd uses reference attributes [12], which we also use in a number of decorators. Its extensions include collection attributes [22] and circular computations [23]. These are built into the JastAdd implementation; there is no user-level mechanism to define similar extensions. As described

\footnotetext{
${ }^{1}$ For the purposes of this paper, we focus on the attribute grammar features of JastAdd, ignoring its support for rewriting trees during evaluation [9].
} 
in Section 4, decorators can be used to define these same features at a higher level. Admittedly, we would not expect users to define relatively complex features like this very often, but building on the high-level framework provided by decorators is likely to be much easier than modifying the underlying implementation of an AG evaluation system. JastAdd is designed to be used in conjunction with hand-written code, particularly using visitors. As such, it provides a way to write traversals that interoperate with declarative attribution. In theory, this facility could be used to implement something similar to decorators, but this would require the addition of generic traversal on top of the Java implementation of trees, essentially duplicating the Stratego platform we use.

\section{Conclusions and Future Work}

We propose decorated attribute grammars as a formalism for application-level extensibility of AG systems. To this end, we have identified primitives for the specification of decorators to define abstract evaluation strategies for attributes. By means of a prototype implementation and by employing decorators in different language engineering applications, we demonstrated the feasibility of using decorators to implement common abstractions over basic attribute grammars. These can be provided in the form of a library, and may be extended with user-defined decorators, where decorator stacking can be applied to reuse existing definitions.

In the future, we would like to explore further applications of decorated attribute grammars, in particular in the domain of implementing domain-specific languages and modular language extensions. For this we want to build upon the rewriting capabilities of the Stratego transformation language, the foundation of ASTER. As such, we aim to take the best of both worlds; rewriting with Stratego and declarative analysis with attribute grammars.

Building on our past experience [16], another application area to which we want to apply ASTER is that of integrated development environments (IDEs). ASTER's performance is already sufficient to be usable, and its demand-driven evaluation further helps interactive application. As such, we would like to employ it as part of an IDE in the future, encapsulating logic for typical editor service components, incremental compilation concerns, and related patterns in decorators.

Acknowledgments. We would like to thank Nicolas Pierron for the discussions on attribute grammar systems and their implementation. This research was supported by NWO projects 638.001.610, MoDSE: Model-Driven Software Evolution, 612.063.512, TFA: Transformations for Abstractions, and 040.11.001, Combining Attribute Grammars and Term Rewriting for Programming Abstractions.

\section{References}

1. Aster project home page, http://strategoxt.org/Stratego/Aster

2. Baars, A., Swierstra, D., Löh, A.: UU AG System User Manual. Department of Computer Science, Utrecht University (September 2003)

3. Bird, R.: Using circular programs to eliminate multiple traversals of data. Acta Informatica 21(3), 239-250 (1984) 
4. Boyland, J.: Descriptional Composition of Compiler Components. PhD thesis (1996)

5. Boyland, J.: Remote attribute grammars. Journal of the ACM (JACM) 52(4), 627-687 (2005)

6. Boyland, J., Graham, S.L.: Composing tree attributions. In: POPL 1994, pp. 375-388. ACM, New York (1994)

7. van den Brand, M.G.J., de Jong, H., Klint, P., Olivier, P.: Efficient annotated terms. Software, Practice \& Experience 30(3), 259-291 (2000)

8. Bravenboer, M., Kalleberg, K.T., Vermaas, R., Visser, E.: Stratego/XT 0.17. A language and toolset for program transformation. Science of Computer Programming 72(1-2), 52-70 (2008)

9. Ekman, T., Hedin, G.: Rewritable reference attributed grammars. In: Odersky, M. (ed.) ECOOP 2004. LNCS, vol. 3086, pp. 144-169. Springer, Heidelberg (2004)

10. Farnum, C.: Pattern-based tree attribution. In: POPL 1992, pp. 211-222 (1992)

11. Gamma, E., Helm, R., Johnson, R., Vlissides, J.: Design patterns: elements of reusable object-oriented software. Addison-Wesley Professional, Reading (1995)

12. Hedin, G.: Reference attributed grammars. Informatica (Slovenia) 24(3), 301-317 (2000)

13. Hedin, G., Magnusson, E.: JastAdd - an aspect-oriented compiler construction system. Science of Computer Programming 47(1), 37-58 (2003)

14. Jalili, F.: A general linear time evaluator for attribute grammars. ACM SIGPLAN Notices 18(9), 35-44 (1983)

15. Kastens, U., Waite, W.M.: Modularity and reusability in attribute grammars. Acta Informatica 31(7), 601-627 (1994)

16. Kats, L.C.L., Kalleberg, K.T., Visser, E.: Generating editors for embedded languages. Integrating SGLR into IMP. In: LDTA 2008 (April 2008)

17. Kats, L.C.L., Sloane, A.M., Visser, E.: Decorated attribute grammars - Attribute evaluation meets strategic programming. Extended technical report TUD-SERG-2008-038a. Software Engineering Research Group, Delft University of Technology (2008), http://swerl.tudelft.nl/bin/view/Main/TechnicalReports\#2008-038

18. Kiczales, G., et al.: Aspect-oriented programming. In: Aksit, M., Matsuoka, S. (eds.) ECOOP 1997. LNCS, vol. 1241, pp. 220-242. Springer, Heidelberg (1997)

19. Knuth, D.E.: Semantics of context-free languages. Math. Syst. Theory 2(2), 127-145 (1968)

20. Laemmel, R., Visser, E., Visser, J.: Strategic programming meets adaptive programming. In: Proceedings of Aspect-Oriented Software Development (AOSD 2003), Boston, USA, pp. 168-177. ACM Press, New York (2003)

21. Lämmel, R.: Typed generic traversal with term rewriting strategies. Journal of Logic and Algebraic Programming 54(1), 1-64 (2003)

22. Magnusson, E., Ekman, T., Hedin, G.: Extending attribute grammars with collection attributes - evaluation and applications. In: Proc. of the Int. Working Conference on Source Code Analysis and Manipulation, pp. 69-80 (2007)

23. Magnusson, E., Hedin, G.: Circular reference attributed grammars - their evaluation and applications. Science of Computer Programming 68(1), 21-37 (2007)

24. de Moor, O., Backhouse, K., Swierstra, S.: First-class attribute grammars. Informatica 24(3), 329-341 (2000)

25. Nilsson-Nyman, E., Ekman, T., Hedin, G., Magnusson, E.: Declarative intraprocedural flow analysis of Java source code. In: LDTA 2008 (2008)

26. Paakki, J.: Attribute grammar paradigms - a high-level methodology in language implementation. ACM Computing Surveys (CSUR) 27(2), 196-255 (1995)

27. van Rossum, G.: Python Reference Manual. iUniverse (2000)

28. Steele, G.: Growing a language. Higher Order Symb. Comp. 12(3), 221-236 (1999)

29. Van Wyk, E., Bodin, D., Gao, J., Krishnan, L.: Silver: an extensible attribute grammar system. In: LDTA 2007. ENTCS, vol. 203, pp. 103-116. Elsevier Science, Amsterdam (2008) 
30. Van Wyk, E., de Moor, O., Backhouse, K., Kwiatkowski, P.: Forwarding in attribute grammars for modular language design. In: Horspool, R.N. (ed.) CC 2002. LNCS, vol. 2304, pp. 128-142. Springer, Heidelberg (2002)

31. Van Wyk, E., Krishnan, L., Bodin, D., Johnson, E.: Adding domain-specific and general purpose language features to Java with the Java language extender. In: Companion to OOPSLA 2006, pp. 728-729. ACM, New York (2006)

32. Visser, E.: Meta-programming with concrete object syntax. In: Batory, D., Consel, C., Taha, W. (eds.) GPCE 2002. LNCS, vol. 2487, pp. 299-315. Springer, Heidelberg (2002)

33. Visser, E., Benaissa, Z.-e.-A., Tolmach, A.: Building program optimizers with rewriting strategies. In: International Conference on Functional Programming (ICFP 1998), pp. 1326. ACM, New York (1998) 